\title{
Geometrical Analysis of the Coffers of the Pantheon's Dome in Rome
}

\author{
Licinia Aliberti ${ }^{1} \cdot$ Miguel Ángel Alonso-Rodríguez ${ }^{1}$
}

Published online: 25 April 2017

(C) Kim Williams Books, Turin 2017

\begin{abstract}
The shape and layout of the coffers of the Pantheon's dome have been studied for centuries. The configuration of the coffers is governed by a complex design that is based on geometric-constructive laws yet to be defined. There is no recent and complete graphic restitution of the Pantheon in scientific publications. In this research, contemporary metric restitution techniques were employed to survey the dome's soffit. The layout of the coffers and their relationships with the spherical surface were analysed according to the performed survey. Through a comparative analysis between the restitution model and the ideal model of the dome's soffit, we can identify possible irregularities and the laws that govern the elaborated design of these elements.
\end{abstract}

Keywords Roman architecture · Pantheon - Coffer layout · Geometric analysis

\section{Introduction}

Roman concrete exhibits unique capabilities in the design and construction of the internal surfaces of vaults. This material offered the possibility to experiment with different geometries. A wide range of dimensions, geometries and building systems applied in different places and conditions have been preserved. Among the different examples of Roman domes, the Pantheon stands as an extraordinary case due to its

Licinia Aliberti

licinia.aliberti@gmail.com

Miguel Ángel Alonso-Rodríguez

miguel.alonso@upm.es

1 UPM, ETSAM, Av. Juan de Herrera, 4, 28040 Madrid, Spain

Birkhäuser 
enormous dimensions, its state of preservation and the complex design, shape and layout of the coffers (Fig. 1).

This present work is part of more extended research on the Pantheon and Roman domes, with a special focus on the internal surfaces of the vaults and the evolution of the construction techniques as these domes became larger. This knowledge establishes the framework in which the geometrical analyses are developed. We analysed a number of Roman domes, visiting many of their sites and studying their construction and the geometry of their inner surfaces (Aliberti 2013).

This exploration of the constructive evolution of methodology and form suggests the presence of recurring elements that we can find in the Pantheon's dome (Gambardella 2002; Lancaster 2005; MacDonald 2002). This great monument presents many questions that remain unanswered. The principal subjects of contemporary research on the building include (1) which construction techniques were used to build such an enormous vault, and (2) how did the Romans control the complex design of the Pantheon's coffers?

There are many hypotheses regarding the initial scaffolding and form-work construction, as it was a complex constructive problem at the time of construction. The scaffolding structure used during the restoration work of 1930 shows the complex solution chosen to reach the surface of the dome (Belardi 2006: 169),

Fig. 1 Photograph of the coffers of the Pantheon's dome

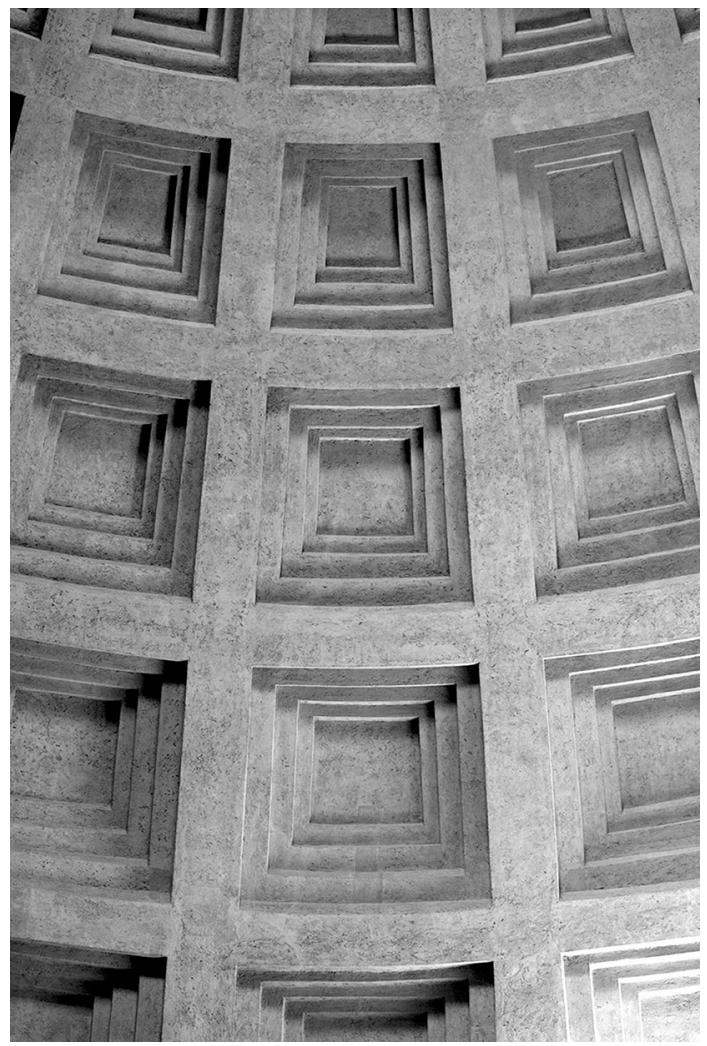


which provides insight to the problem of assessing the initial construction of the vault. We believe that studying the design of the coffers could shed light on this difficult condition.

The Pantheon has been graphically represented for centuries because of the prominence of the monument, its amazing features and its perfect state of preservation. However, in recent scientific publications, no complete graphical restitution of the dome of the Pantheon exists as the surveys used by Valenti (2009) and Belardi (2006) are not yet published; therefore, some authors still use old documents to formulate new hypotheses. For example, Waddell (2008) used measurements documented by Piranesi in the seventeenth century to formulate his hypothesis about the distribution of coffers. In the Nexus Network Journal, Fernandez-Cabo (2013) stressed this evidence and eventually used the drawings of Rondelet (1860) to carry out his research.

Here, we aim to offer some documentation and data based on a contemporary double survey that we developed using digital photogrammetry and a three-dimensional laser scan (Fig. 2). A current architectural survey of the interior of the dome provides the basis to develop this work and can generate useful material for future studies.

\section{Survey Model and Ideal Model of the Dome's Surface}

The geometrical analyses that we propose are based on a comparison between the survey model and the ideal model. The survey points determine the creation of a regular model defined by clarifying geometrical laws with a rigorous average calculation. The direct comparison between the individual positions of the raised points and the ideal model contributes to understanding the building. The comparison detects irregularities or deformities where they exist and provides objective and quantifiable data. Vector-drawing graphical tools and a system of calculations to objectively quantify the outcomes have been applied to develop the analysis.

The first step in building the ideal model is to construct the average sphere based on the coordinates of the survey points. Then, an accurate division into 28 equal

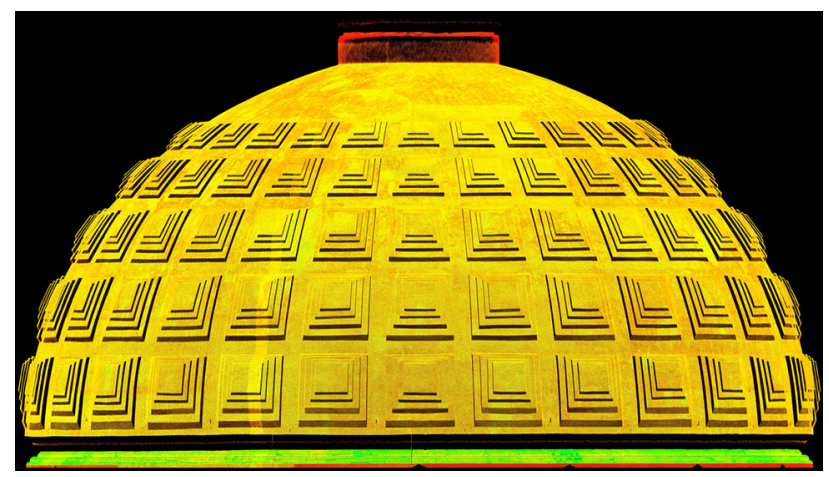

Fig. 2 View of the point cloud obtained with a three-dimensional laser scan 
sectors is obtained. This first approach to the ideal model is useful for studying the inner surface of the dome and the general distribution of the coffers (Fig. 3).

A more complex procedure is required to obtain the construction of the ideal sector of the coffers. A previous study and a detailed geometrical analysis based on the survey model are needed. The average measurements obtained and the alignments of the vertex of coffers contribute to reconstructing the ideal sector and complete the building of the ideal model. The construction of an ideal sphere considers the points of a photogrammetric model and results in an average radius of $22.03 \mathrm{~m}$. The diameter of this calculus matches the result obtained by the point cloud management program. In this way, we obtain a double verification of the accuracy of the average value.

The first comparison between the survey model and the ideal model is represented by the intersection of the two surfaces (Fig. 4). A area of depression is detected along the North-South axis. A similar finding was obtained by Scherer (2009: 220-221). Identifications of deformed areas are useful data, as they may be detected in the shape and distribution of coffers. To determine the possible deformation of the dome, we calculated the distance from the survey points to the ideal surface. The data of the outcome show a $10 \mathrm{~cm}$ tolerance in $72.5 \%$ of the points, whereas $21 \%$ are under $15 \mathrm{~cm}$, and $6.5 \%$ are over this measure. The results indicate that a large number of points are very close to the spherical surface.

According to the classic descriptions of the Pantheon, the centre of the dome resides at the plane of the cornice between the drum and the dome. The present work, like Fine Licht (1968), Pelletti (1989) and Graßhoff et al. (2006), confirms the existence of a gap between the plane of the cornice and the centre of the sphere. To reconstruct the cornice line, we applied an average calculation to the survey points.

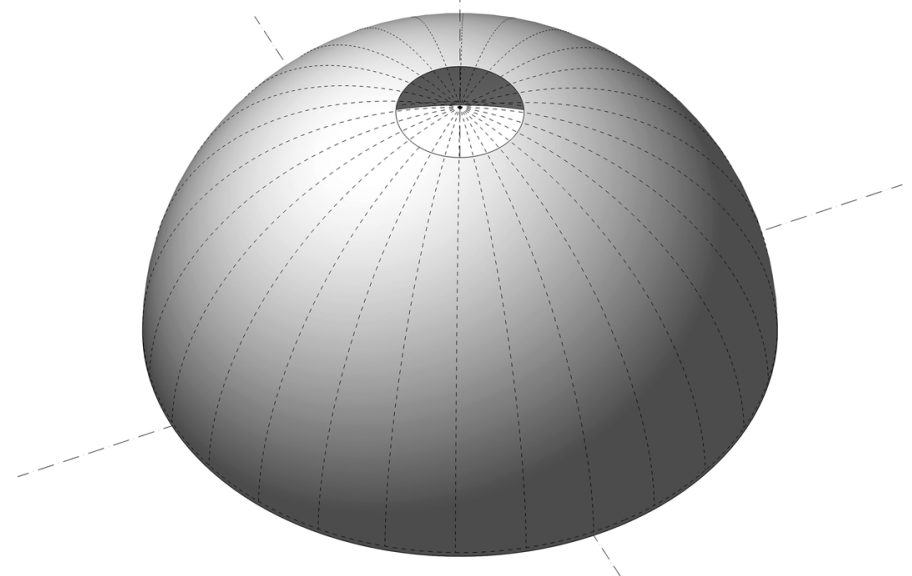

Fig. 3 View of the first approach to the ideal model 


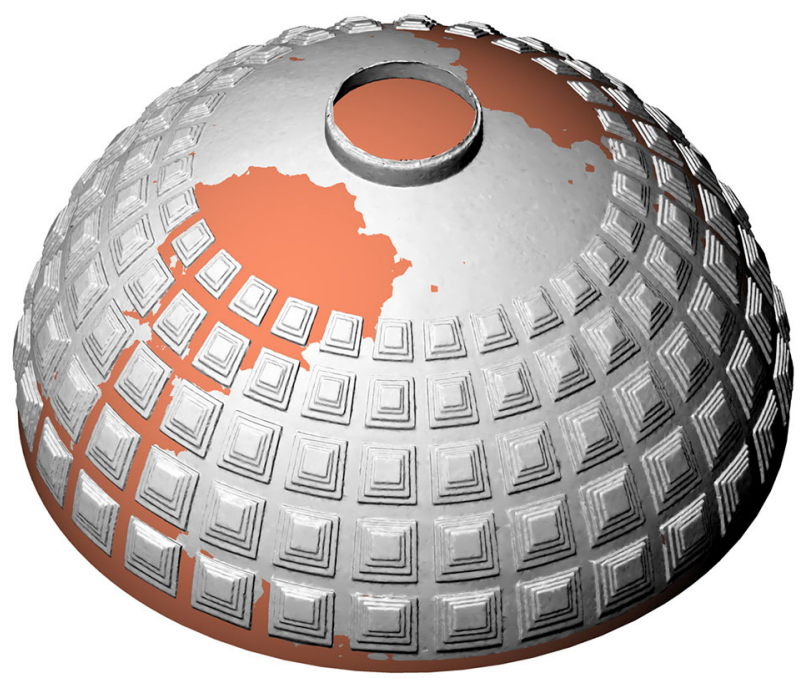

Fig. 4 View of the comparison between the survey model (grey) and the ideal model (red) (colour figure online)

The position of the centre of the ideal sphere was calculated at $-0.37 \mathrm{~m}$ behind the cornice line.

One of the factors that has been taken into account when analysing the dimensions of the Pantheon's dome is the building's expansion. Concrete expands, and the average coefficient of thermal expansion is $10^{-5}{ }^{\circ} \mathrm{C}^{-1}$. Test results show that this coefficient can change in a relatively high proportion (on the order of $\pm 30 \%$ ) depending on the nature of the concrete, sand and gravel, dosage, hygrometry and dimensions of the sections. The average value considered above results in a length variation of $8.7 \mathrm{~mm}$ for an initial length of $44.06 \mathrm{~m}$ (the approximated value of the Pantheon's dome) and a temperature difference of $20{ }^{\circ} \mathrm{C}$ with regard to the average temperature. This change in length would occur when the corresponding thermal oscillation affected the entire volume of concrete. This situation seems unlikely due to the thermal inertia of the building, which is certainly very high because of the width of its walls and sections. For these reasons, we consider that it is reasonable not to include thermal expansion in our analysis.

\section{The Shape and Layout of the Coffers}

The research is conducted in two phases. It begins with a study of the general layout of the coffers relative to the sphere, together with horizontal and vertical orientations of the 28 sectors and 5 levels. Subsequently, a closer analysis of the shape and geometry of the coffers is proposed, providing a set of metric data that have been processed on the basis of the results from the previous survey and proposing design criteria for the construction of the ideal sector of the coffers. The following factors have been considered. 
General arrangement:

- Division of the circumference into 28 equal parts;

- Location of the axes of the bisectors of the sectors;

- Main axis accuracy;

- Average circumferences passing through the vertex of the coffers: concentricity and orientation;

- Vertical external lines of the convergence of the coffers.

Shape and geometry:

- Analysis of surface geometry;

- Dimensions study: calculation of averages and study of diminution of dimensions;

- Vertical stripes between sectors and horizontal stripes between levels;

- Convergence of transition surface directions;

- Comparative study of the perspective projection of the coffers;

- Proportion of external and inner shapes of coffer levels.

\section{General Arrangement}

The division of the hemisphere into 28 equal parts was difficult to obtain at the time of construction. The division of a quarter of the circumference into 7 equal parts was a complicated geometric problem (Martines 1989). Moreover, the setting out on site was especially difficult at the location of the cornice of the vault. The choice of this complex division may be associated with cultural and symbolic reasons (Lucchini 1996: 109; Wilson Jones 2000: 183) or with the special proportions of the coffers adapting to the spherical surface (Saalman 1988: 121).

Pelletti (1989: 17) described the ancient method of dividing a circumference using strings, but stated that it would not have been possible to use this method for the Pantheon's dome due to the scope of the project and the presence of scaffolding structures. Taylor (2006: 199) proposed the hypothesis that the division was made on the floor and then disassembled and reassembled at the site of the cornice line. This system allowed for a reduction of possible mistakes, but it would also stop construction for a considerable period of time. Waddell (2008: 84) put forward a theory about the mathematical division of the circumference into 28 equal parts by using a specific number of Roman feet.

According to the calculations of the present study, the accuracy of the radial distribution of the coffers is surprising (Fig. 5). When observing deviations in the plan between the bisectors of the coffer sectors and the axes that theoretically divide the circumference into 28 equal parts, the average recorded deviation barely reaches $0^{\circ} 36^{\prime}$ (Table 1). The angles between vertical axes of the coffer sectors are extremely close to $12^{\circ} 50^{\prime}$, which is equivalent to the subdivision of the $360^{\circ}$ ideal model into 28 equal parts.

We noted that the axis of the coffer sector situated opposite the entry portal is parallel to the North-South axis at a distance of $15 \mathrm{~cm}$. This detail may indicate that 


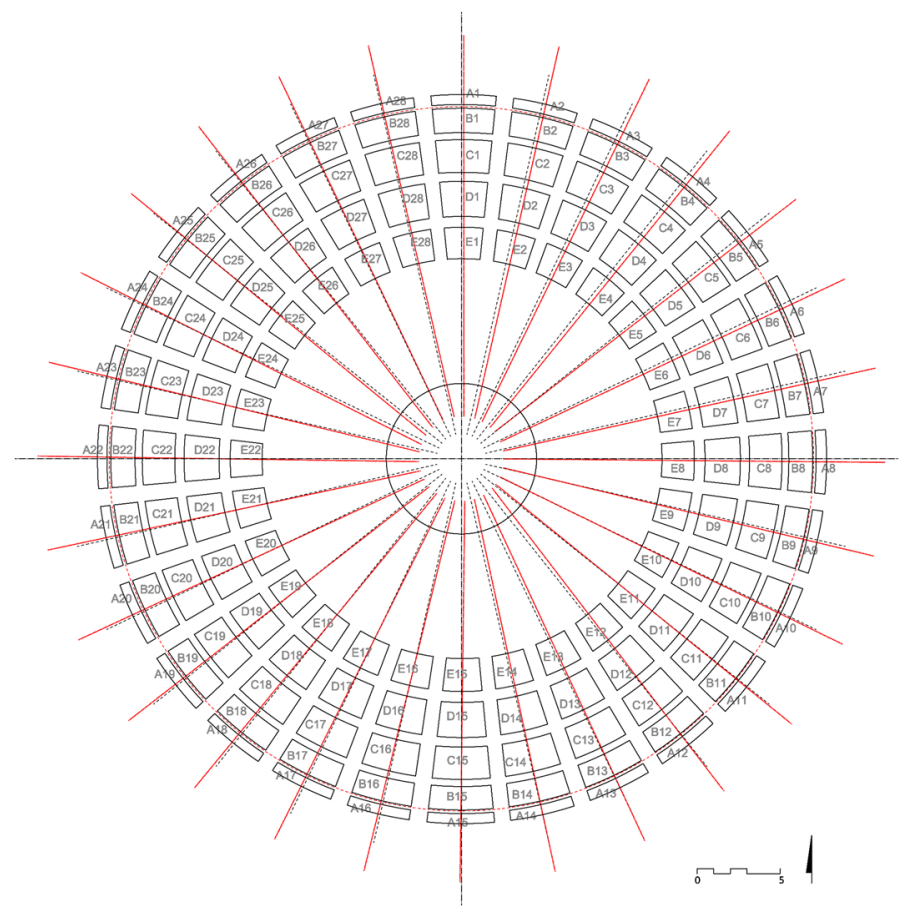

Fig. 5 General arrangement: analysis of the accuracy of the main axes and of the distribution of bisectors of the sectors

Table 1 Angles between the bisectors of the coffer sectors

\begin{tabular}{llllllll}
\hline Quadrants & \multicolumn{2}{l}{ Angles between bisectors of coffer sectors (ideal division of $360^{\circ}$ into 28 equal } \\
& parts $=12.85^{\circ}$ ) \\
\hline I & $12.80^{\circ}$ & $13.23^{\circ}$ & $12.91^{\circ}$ & $13.42^{\circ}$ & $12.37^{\circ}$ & $12.76^{\circ}$ & $13.06^{\circ}$ \\
II & $12.86^{\circ}$ & $12.64^{\circ}$ & $12.98^{\circ}$ & $13.56^{\circ}$ & $12.44^{\circ}$ & $12.75^{\circ}$ & $12.95^{\circ}$ \\
III & $13.07^{\circ}$ & $12.74^{\circ}$ & $12.93^{\circ}$ & $12.68^{\circ}$ & $12.95^{\circ}$ & $12.93^{\circ}$ & $12.83^{\circ}$ \\
IV & $12.75^{\circ}$ & $13.17^{\circ}$ & $12.59^{\circ}$ & $12.78^{\circ}$ & $12.34^{\circ}$ & $12.52^{\circ}$ & $12.98^{\circ}$ \\
\hline
\end{tabular}

the division of the circumference into 28 equal parts might have been started or completed with this sector.

If we observe the alignment between the sectors of quadrants I and III, we can see that the six opposing coffer sectors are perfectly aligned. However, the sectors of quadrants II and IV are not aligned with their opposites. The maximum divergence is registered in the two sectors of quadrants II and IV, where the bisectors reach a distance of $39 \mathrm{~cm}$ from the centre of the ideal sphere.

We continued the observation of the general arrangement by studying the main axes in plan view. 
The accuracy of the orientation of the North-South axis stands out for its precision if we compare it with other Roman buildings with similar dimensions. For example, the Coliseum shows a more pronounced gap in the orthogonal direction of the main axis (Pelletti 1989: 15). In studying the division of the circumference into four quadrants, it is evident that the longitudinal and transversal axes are approximately orthogonal. However, we can also observe that the four lines that result slightly diverge from the centre of the ideal sphere. The East transversal axis shows a distance from the centre of the sphere of approximately $16 \mathrm{~cm}$ and the West transversal axis has a slightly greater value. The two parts show a minimum deviation from the perfect orthogonality: $90^{\circ} 50^{\prime}$ between the East transversal axis and the longitudinal axis; $90^{\circ} 32^{\prime}$ between the West transversal axis and the longitudinal axis.

Another topic included in this research is the verification of the horizontality of the different coffer levels. The average circumferences following the points defined by horizontal edges generally present a slight slope relative to the horizontal plane, with an average value of approximately $0^{\circ} 19^{\prime}$ (Fig. 6). All maximum slope lines of the planes defined by the circumferences are oriented northwest/southeast.

The upper circumference of the last level of the coffers shows a greater inclination than the average value, and it is directed towards the North direction. This difference may evidence an actual defect in the design construction if compared with the other levels, even if the value is very slight $\left(0^{\circ} 27^{\prime}\right.$ with the

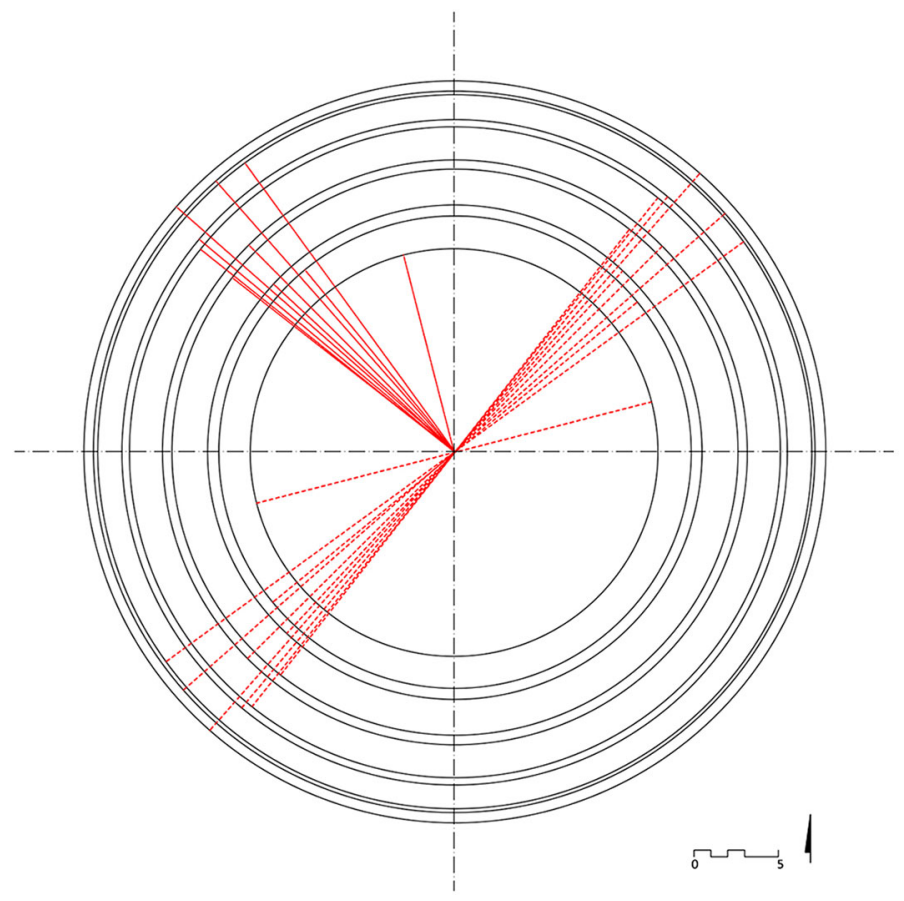

Fig. 6 Average circumferences passing through the external points of the coffers: analysis of horizontality and concentricity 
horizontal plane). The average circumferences are approximately concentric. The main distance between the centre of the circumferences and the centre of the ideal sphere is only $7 \mathrm{~cm}$. These data provide new affirmation of the incredible accuracy of the vault to the ideal model, and also confirms the uniformity of the general distribution of the coffers.

By studying the shape of the vertical sectors, it can be seen that the curves representing the alignments of the external points of the coffers do not converge towards the sphere's pole, as might be assumed (Fig. 7). Based on our study of the bisectors of the coffers, the sectors tend to converge with high approximation towards the centre of the sphere. The external limits of the coffers might have been traced from them according to a system based on the accuracy of the bisector design.

The extension of these external lines of each sector generates a vertex at a certain distance from the centre of the sphere. The locations of these points do not approximate a circumference but rather show evident irregularities. If we attempt to establish a relation between this fact and the analysis of the dome surface, it appears that the arrangement of the vertexes somehow reflects the presence of deformed areas in the North-South zone of the dome. In fact, the vertexes that present the greatest distance from the centre are located in this area.

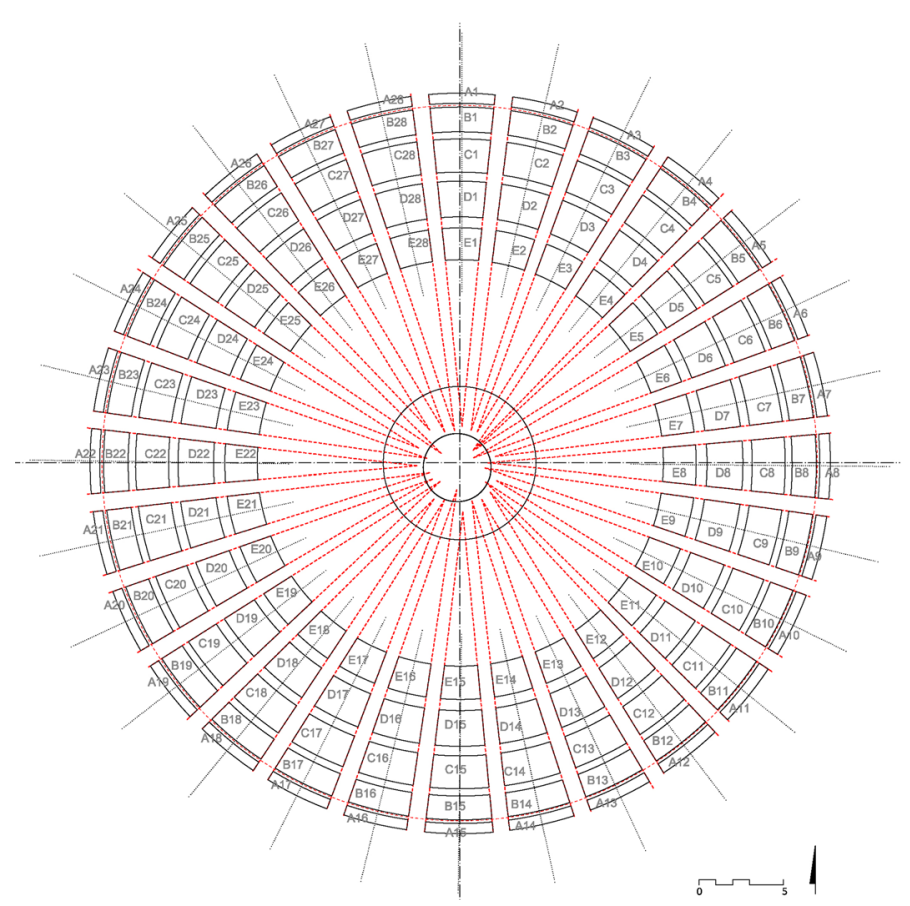

Fig. 7 Alignments of the external edges of the coffers showing that they do not converge in the upper pole of the sphere 


\section{Shape and Geometry}

The continuous reduction of the dimensions and variations in coffer shape, when adapted to the curvature of the sphere, result in a harmonious design of the dome soffit. The visual perception of the coffers is influenced by the inner surface proportions and the diverse directions of the transition surfaces.

The Romans used coffers in barrel vaults, but there are few surviving examples of double-curvature surfaces. One of the most ancient examples is the vault of the sanctuary of Fortuna Primigenia, which dates from the end of the second century B.C. (Lugli 1957: Tav. CCIX). In this case, the coffers have a trapezoidal shape and allow a complete perspective vision adapting to the vault surface.

In the Thermal Baths of Trajan and in the Pantheon, we find the first preserved examples of coffers adapted to a spherical surface (Waddell 2008: 58). Fine Licht (1968: 276) and Wilson Jones (2000: 192) noted the relevance of the perspective design and radial distribution of the coffers of the East room of the Baths, while the remaining structures cannot be evaluated due to their poor state of conservation.

In the Pantheon the coffers that appear square to the eye actually have a trapezoidal shape. The control of visual effects may be directed to establish a connection between coffer distribution and the pavement grid, which is composed of

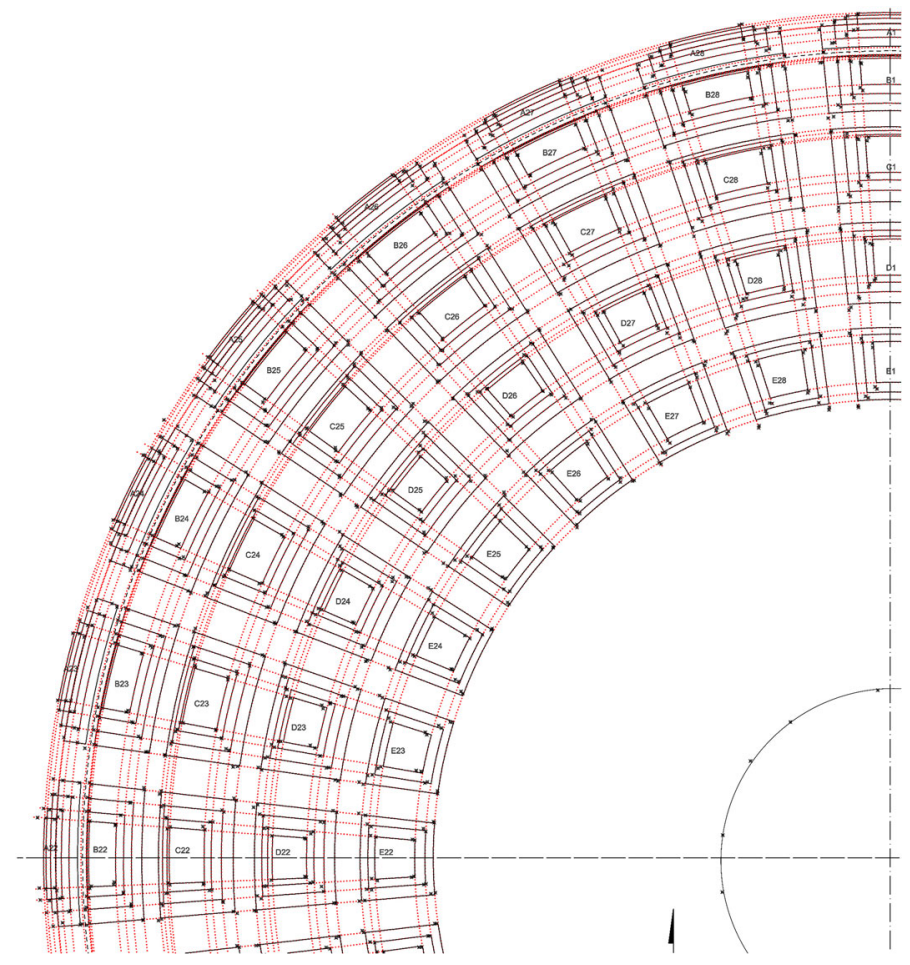

Fig. 8 Arrangement of coffers. Study of the alignment through calculated average of the positions of the survey points 
large squares. The dimensions of the floor squares are visually similar to the image of the dome coffers (Waddell 2008: 85) (Fig. 8).

Many authors, including Fine Licht (1968: 140) and Waddell (2008: 85), have stated that the coffers of the Pantheon's dome do not play a constructive or structural role but are merely decorative elements. Their function is primarily related to visual perception, which may justify their complex design.

We began the analysis by studying whether the different surfaces making up the coffers are spherical or flat (Fig. 9). An ideal flat surface was created with successive calculations on each of the intermediate and deepest levels of one sector of coffers. The distances of the raised point from this flat average surface were minimal $(2-3 \mathrm{~cm})$. It can thus be deduced that the intermediate and the deepest levels do not adapt to a spherical surface but tend to be flat, which would undoubtedly make it easier to control the formwork.

One of the dome quadrants was carefully studied: dimensions of six sectors of coffers were recorded, and the data were processed through a calculation of averages to obtain reference values of their measurements. We drew an orthogonal projection of each coffer of the second sector of the North-East quadrant. Even if these projections are orthogonal, they reduce the actual length of the curves due to their restitution onto a plane. Moreover, we proposed another calculation of the

Fig. 9 Photograph of the internal surfaces of the coffers

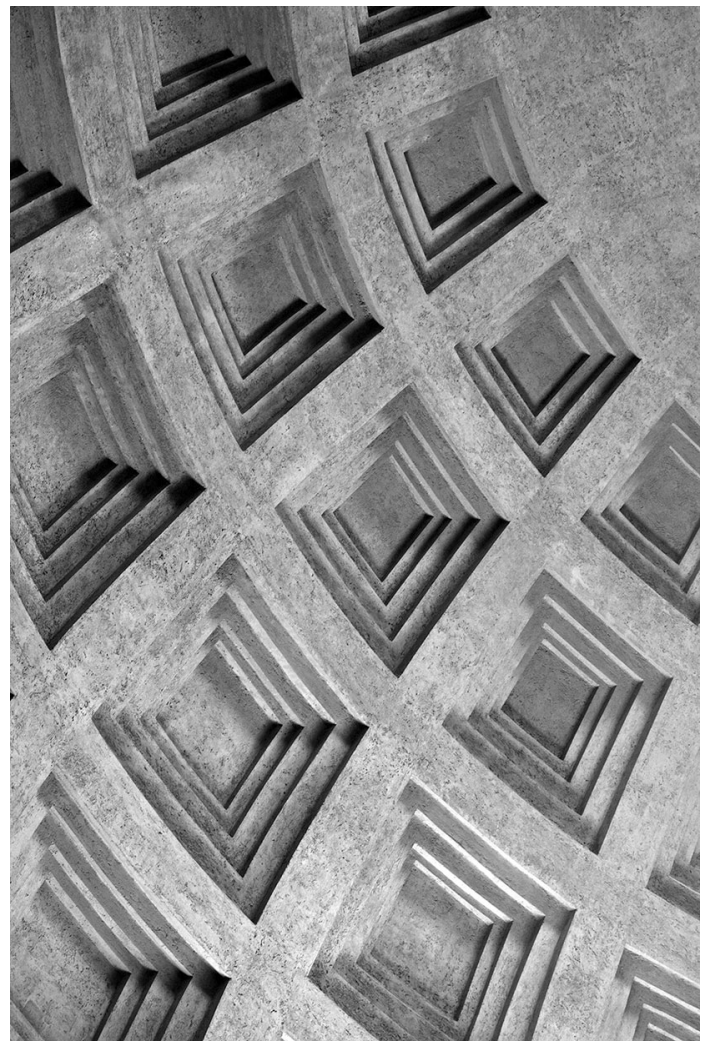


Table 2 North-East quadrant

\begin{tabular}{llllll}
\hline & A & B & C & D & E \\
\hline Bottom width & 3.88 & 3.74 & 3.39 & 2.92 & 2.38 \\
Upper width & 3.77 & 3.48 & 3.02 & 2.50 & 2.02 \\
Height & 4.08 & 3.79 & 3.52 & 3.05 & 2.39 \\
\hline
\end{tabular}

Average absolute values of sectors 2-6

length of the arcs that defines the external edges of the coffers. The values of sectors 2, 3, 4, 5 and 6 of the North-East quadrant were registered and used to obtain the average values (Table 2 ).

The results show a gradual decrease of the length of the vertical arcs of the coffers. The average values from the first level (A) to the last level generated a numerical progression:

\begin{tabular}{llll}
\hline 0.93 & 0.86 & 0.75 & 0.60 \\
\hline (B:A) & (C:A) & (D:A) & (E:A) \\
\hline
\end{tabular}

This progression increased with the upper levels and was related to the different perception of the curvature of the vault. The last level of coffers shows the greatest difference with the previous level.

The width of the coffers also progressively decreased as they progressed towards the upper levels. The difference between the upper and lower edges of each coffer increased in the higher levels, reflecting the necessity to adapt to the spherical curvature. The upper width of the last coffer was approximately half of the lower length of the coffers at the first level. The analysis shows that the coffer sector of the North-South axis opposite from the entrance portal has a major difference in width (the coffer of the first level of this sector, named A1, measures $4.00 \mathrm{~m}$ in its lower part, whereas the rest of coffers measure as follows: A2, $3.90 \mathrm{~m}$; A3, $3.94 \mathrm{~m}$; A4, $3.86 \mathrm{~m}$; A5, $3.83 \mathrm{~m}$; A6, $3.86 \mathrm{~m}$; A7, $3.85 \mathrm{~m}$; A8, $3.92 \mathrm{~m}$ ). This difference has an impact on the radial arrangement of the coffers and generates the divergence of the bisector that was registered by the comparison of the survey model and the ideal model. Moreover, as in our previous study (Aliberti et al. 2014), the bisector of this sector shows a gap with the North-South axis. This divergence from the average value was distributed evenly between the six sectors of the second quadrant in order to generate a uniform image of the soffit frame.

The results suggest that the division of the circumference into 28 equal parts could not be controlled by rigid geometrical construction; the layout of the coffers was adapted to the slight divergences of the North-South sector to determine a uniform distribution using an empirical method. The setting-out on site may have been performed through a practical-constructive technique based on experimentation more than complex geometrical laws. 
The depth of the intermediate surfaces changed at different coffer levels. Using the section passing through the vertical axis of the coffer sector, we studied the second sector of the North-East quadrant (Fig. 10). As we can see in Table 3, there is a small and gradual decrease in depth from the first level to the fifth level. The slight divergence recorded between the first three internal surfaces measures a maximum of $4 \mathrm{~cm}$. It is evident that the greatest decrease in depth appears in the last surface. This fourth surface disappears in the upper level of the coffers; therefore, the third surface follows a different design.

Unlike the coffer width and height variations, the horizontal bands separating the coffers have very similar dimensions. When calculating these values as arcs of a sphere, the average value is 0.84 , with variations of a few centimetres between zones. This regularity is also reflected in the separation between the vertical sectors of the coffers. Based on the study of the North-East quadrant, the average distance between the lowest external points of the first level is $1.02 \mathrm{~m}$, which becomes 0.67 between the upper external points of the last level. According to these data, the meridian rib is reduced to almost half of its width from beginning to end, a fact that is not perceived from the overall view of the dome, where these strips appear to have almost uniform dimensions. We used these studies on the average measurement of coffers and their distribution as a first metrical reference to build the complete ideal model.

In analysing the direction of the transition surfaces between the different levels, the radial tracings were identified, and other tracings approximately converged into certain reference points outside the coffers (Fig. 11).
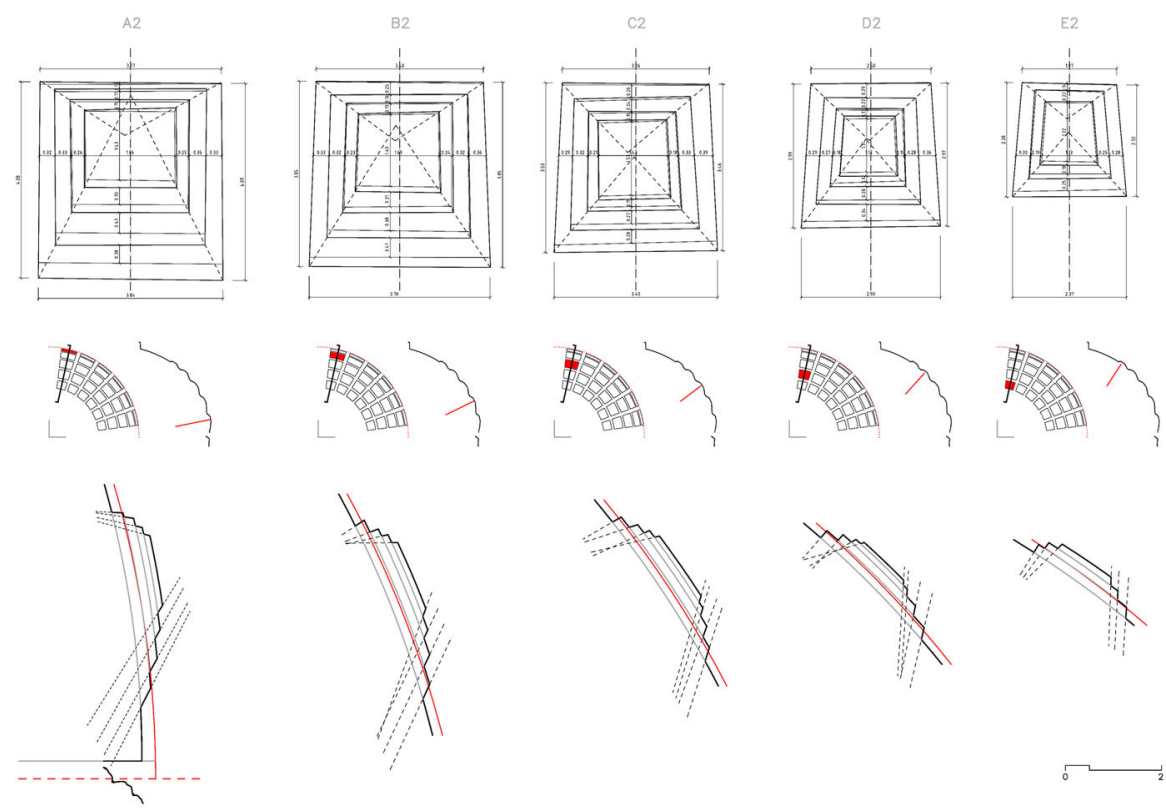

Fig. 10 Coffer dimensions. Vertical section and orthogonal projection of the coffers of sector 2 of the North-East quadrant 
Table 3 North-East quadrant

\begin{tabular}{llllll}
\hline & A2 & B2 & C2 & D2 & E2 \\
\hline $1^{\circ}$ & 0.23 & 0.22 & 0.21 & 0.20 & 0.19 \\
$2^{\circ}$ & 0.19 & 0.18 & 0.18 & 0.16 & 0.15 \\
$3^{\circ}$ & 0.13 & 0.13 & 0.12 & 0.11 & 0.14 \\
$4^{\circ}$ & 0.12 & 0.12 & 0.10 & 0.07 & - \\
\hline
\end{tabular}

Sector 2: depth of intermediate surfaces $(\mathrm{m})$

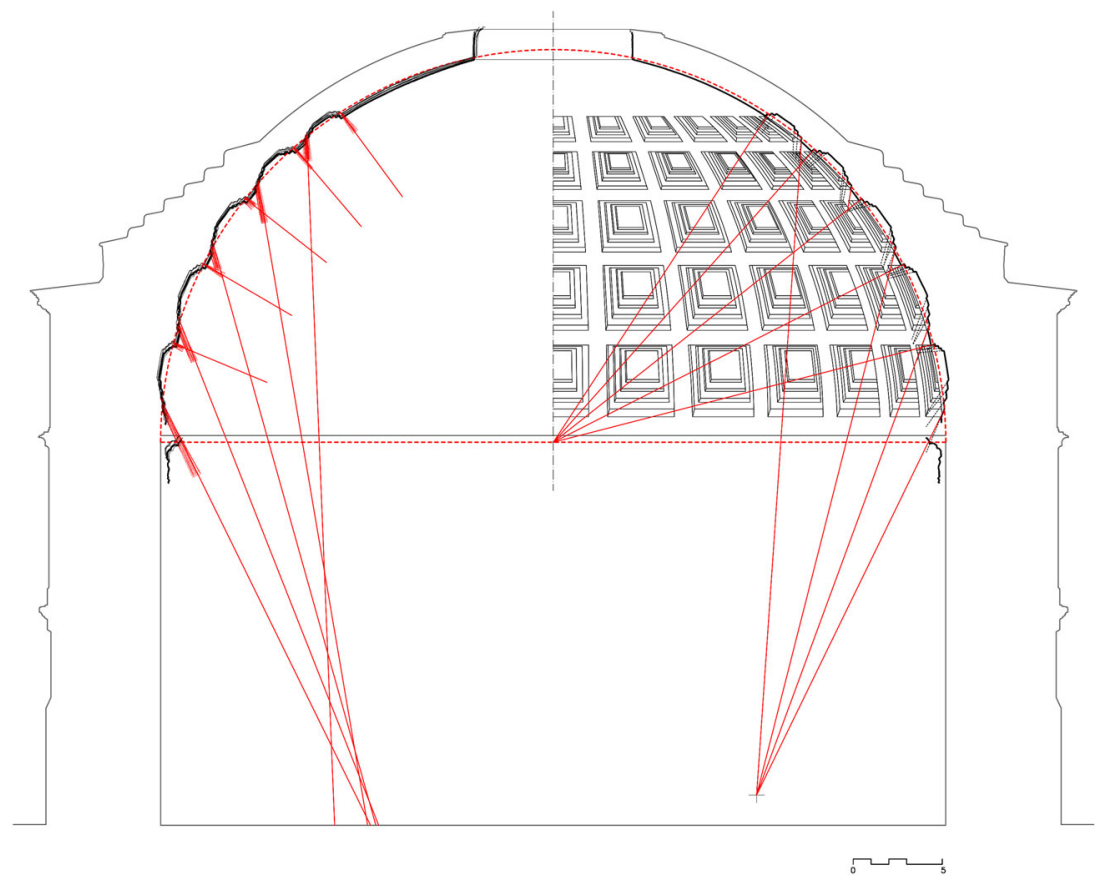

Fig. 11 Study of the direction of the transition surfaces: average calculation applied to the rotation of the radial sections and hypothesis of ideal alignment

These connecting planes are much shorter than the dimensions of the entire dome; therefore, it is difficult to provide an exact definition of their directions. Nevertheless, we can note that the upper connecting surfaces appear to be orthogonal to the spherical surface of the dome. The inferior transition surfaces present a greater inclination in order to open the visual field and leave every edge of the coffers visible.

We interpreted these convergences in the construction of the ideal model (Fig. 12). Here, the upper connecting surfaces were built as radial, and the inferior ones converged to a point at a certain distance from the centre. Although the constructed object is not as exact as the ideal model, these convergences indicate 


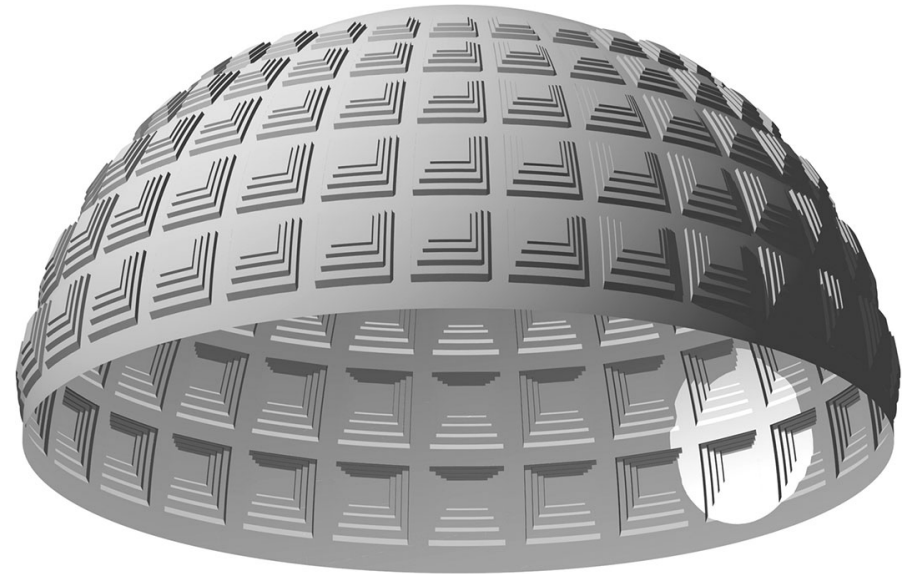

Fig. 12 View of the complete ideal model as result of the geometrical analysis

that there is a circular zone where a viewer can see all the inferior edges of the coffers and almost all the upper edges.

In studying this subject, we should consider that the results are based on the current state of the coffers registered by the point cloud. They may have been slightly modified by the cladding restoration works of Terenzio (1933) in the 1930s and more recently of Belardi (2006), especially if we refer to the smallest surfaces.

The coffer arrangement allows complete visibility from the centre of the floor. This observation has been verified through the study of the point cloud generated from the centre of the room without considering scannings performed on the transversal and longitudinal axes. This unique point cloud perfectly describes every part of the coffers and their complex surfaces without detecting any blind areas. The present research, performed with sophisticated devices and software, verifies the ambitious work of the Roman builders based on empirical knowledge of perception and geometry and carried out with the use of ancient tools.

To perform specific studies of the coffer design, a set of alignments was drawn employing tools that apply average calculations. The drawing of the diagonal lines connecting the external vertices of each coffer's transition surface was uniformly repeated in all of the sectors (Fig. 13). There was a progressive variation in the inclination of the diagonals, which in the first levels clearly pointed towards the upper part of the coffers and gradually declined in the last levels.

To appreciate the perspective attributes of the coffers, a series of projections was performed from different points of view. By comparing different perspective views, it can be directly noted how the perception of these elements slowly changes.

We began the study by generating a frontal perspective view of each coffer of the second sector of the North-East quadrant. The point of view was located in the centre of the room and at a man's height of $1.70 \mathrm{~m}$. It is evident that this perspective view reproduces an almost square image, and the coffer surfaces seem to be regular and of the same dimensions. The perceptive differences of the coffer parts are not visible from the centre of the room. 


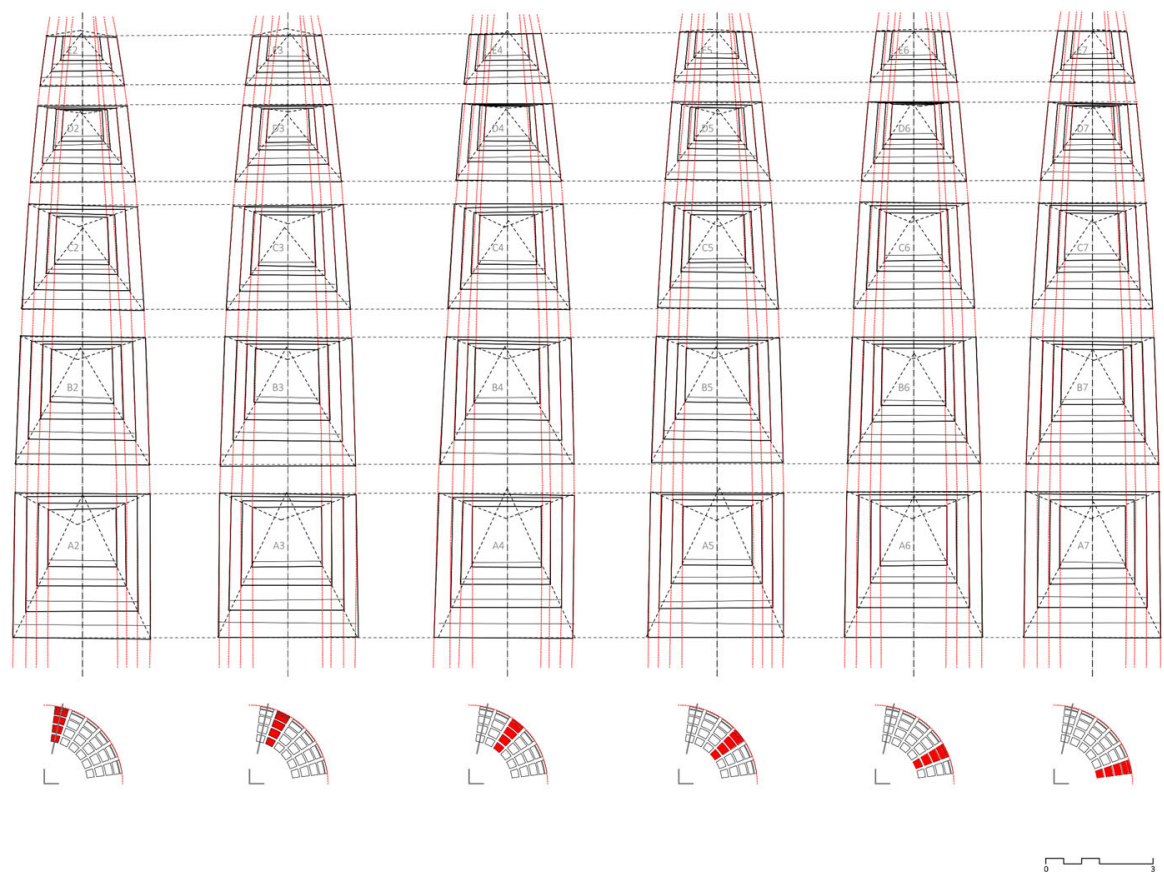

Fig. 13 Elevation of each coffer sector of the North-East quadrant. Study of alignment and proportions

Each coffer generates a different image when the inclination of the perspective axis moves, thus showing its trapezoidal shape. Creating the image of a pure shape, such as a square, is part of the search for regular forms present in the classical Roman culture (Fernández-Cabo 2013: 543). This image of a square may be associated with the external shape of the coffers, while the inner levels show some differences (Fig. 14).

If we approximate the form of the inner levels of the coffers to rectangles, it is evident that their proportions change from the horizontal to the vertical. This change is probably a result of two conditions of coffer design: the aim to preserve the vertical alignment of the sector in the width of both the external and internal levels; and the need for greater alteration of the lowest coffers to control the perspective view from the centre of the room. The lowest connecting planes in the first two levels of the coffers are larger than in the upper levels and the inner figure is thus a horizontal rectangle. From the third level to the fifth, the inner figure is a vertical rectangle. Moreover, the differences between the second and the third level are greater than between the rest of the levels, so the transition seems not to be completely uniform. In any case, it is highly relevant that these rectangles with different proportions are approximately centred in a perspective view from the centre of the room.

Based on these studies, we can state that the design of the inner levels of the coffers seems not to depend on the projection from a hypothetical point, in contrast with some scientific studies. We consider that from the point of view of 


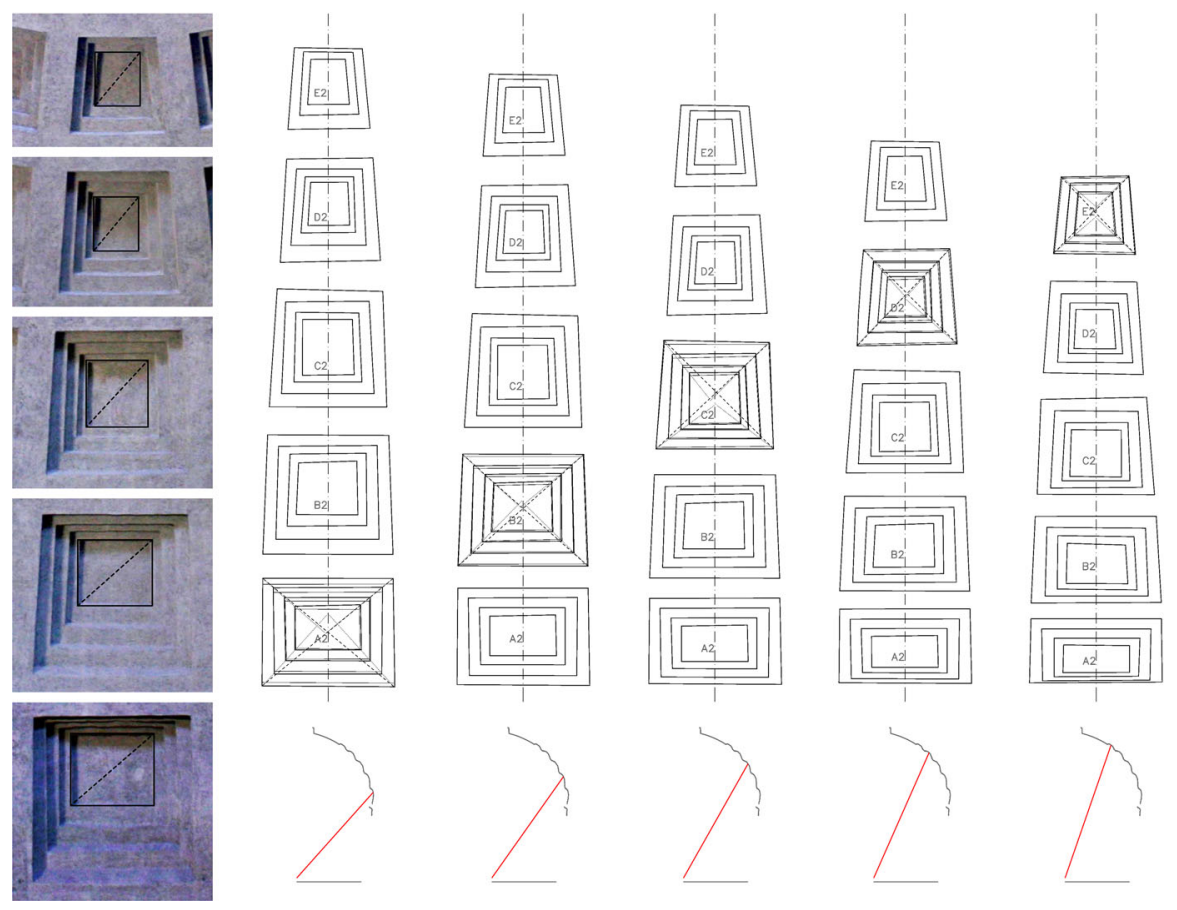

Fig. 14 Study of the proportions of the inner levels applied on the survey model orthogonal views and perspective projection from the centre of the room of the coffers of the North-East quadrant

construction, such a dependence would be very difficult to achieve due to the great distance and the height of the dome from the floor. Furthermore, as far as we currently know, in ancient times there was no knowledge of the perspective method or of spherical geometry.

\section{Conclusions}

This study provides a detailed documentation of the general layout, shape and dimensions of the coffers of the Pantheon's dome and presents different geometrical analyses of specific arguments. The use of contemporary methods of architectural survey assures the correct graphical documentation of the dome in its current state of conservation. Moreover, the use of average calculations is an important tool in this research. A comparison between the ideal and survey models brings to light the surprising uniformity in the distribution of the coffers. At the same time, irregularities are highlighted, and the shape and general arrangement of the coffers is related to the spherical geometry of the soffit.

The analyses developed led to new results in the study of the vault. Some insights involve the general arrangement of the coffers. We can state that the general layout of the 28 sectors of coffers is approximately uniform, but we must stress the special 
evidence of the irregularity of the sector situated on the North-South axis and the consequent distribution of the sectors of the North-East quadrant. This fact may indicate that the distribution of the sectors was adjusted empirically to compensate for the irregular position of the coffer sector situated on the North-South axis.

Another result regarding the general distribution is the analysis of the external lines of the coffer sectors, which appear to converge to the upper pole of the sphere but intersect before this point, reflecting some irregularities. They seem to be traced from the bisectors of coffer sectors that determine the general layout of the coffers.

Other results regard coffer shape and geometry. We consider especially interesting the study of the dimensions of the coffers and their progressive transformation by adapting their dimensions to the spherical surface. The analysis of connecting planes makes it possible to identify the radial direction of the upper transition surfaces and the approximate convergence of the inferior transition surfaces to a point at a certain distance from the centre of the space. We can confirm that a viewer can see a complete view of the coffers from the centre of the space: he can see all the lowest inner edges and many of the upper edges of the coffers from a circular zone at a certain distance from the centre, and he will have a reduced and changing vision of the interior of the coffers from the area next to the circular wall.

Regarding the coffer shape, we propose the hypothesis that Roman constructors designed the external form of the coffers with a square proportion based on the near equivalence between the lowest edge and the vertical edge, adapting to the vertical alignment of the external lines. The more complex design of the inner levels may be the result of two conditions: the vertical alignment of the coffers and the alteration of the lowest parts of the coffers to correct the perspective perception. The resulting shape is different from the usual theoretical image of coffers. The external shape can be approximated as a square, but the inner levels have different proportions and change from horizontal to vertical rectangles. The control of perspective correction was inexact and may not have been generated by a difficult process using a projection point. However, the surprising ability to centre these inner figures within the external shape of the coffers, basing the design on the view from the centre of the space, remains a complicated research topic.

The complex design of the coffers of the Pantheon's dome has yet to be analysed and understood in all its details. This present study provides some new information and contributions to the general research with the aim of offering useful material for future studies.

Acknowledgements We wish to specifically thank the Arch. Giovanni Belardi of the Soprintendenza ai Beni Architettonici di Roma and Professor Marco Canciani of the University of Roma Tre for their contributions in allowing the survey of the Pantheon's vault. All the images shown in this work are by the authors.

\section{References}

Aliberti, Licinia, Miguel Ángel Alonso-Rodríguez and Marco Canciani. 2014. New contributions on the dome of the Pantheon in Rome: comparison between the ideal model and the survey model. ISPRS Archives WG V/4, CIPA 3D-Arch 2015 (XL-5/W4): 291-297. 
Aliberti, Licinia. 2013. Cúpulas clásicas romanas: geometría y construcción. In: VIII Congreso Nacional de Historia de la Construcción, 13-22.

Belardi, Giovanni. 2006. Il Pantheon. Storia, tecnica e restauro. Viterbo: BetaGamma.

Fernández-Cabo, Miguel Carlos. 2013. Analysis of different hypotheses about the geometric pattern of the Pantheon's coffered dome. Nexus Network Journal 15(3):527-47.

Fine Licht, Kjeld de. 1968. The Rotunda in Rome. A study on Hadrian's Pantheon. Copenhagen: Nordisk Forlag.

Gambardella, Alfonso. 2002. Dal Pantheon a Brunelleschi. Architettura, costruzione, tecnica. Napoli: Edizioni Scientifiche Italiane.

Graßhoff, Gerd, Michael Heinzelmann and Marcus Wäfler, eds. 2006. The Pantheon in Rome: Contributions to the conference (Bern, November 9-12, 2006). Bern: Bern Studies in the History and Philosophy of Science.

Lancaster, Lynne. 2005. Concrete Vaulted Construction in Imperial Rome. Innovations in Context. Cambridge: Cambridge University Press.

Lucchini, Flaminio. 1996. Pantheon. Roma: Carocci.

Lugli, Giuseppe. 1957. La tecnica edilizia romana con particolare riguardo a Roma e Lazio. Roma: Giovanni Bardi Editore.

MacDonald, William L. 2002. The Pantheon: Design, meaning and progeny. Harvard: Harvard University Press.

Martines, Giangiacomo. 1989. Argomenti di geometria antica a proposito del Pantheon. Quaderni dell'Istituto Di Storia dell'Architettura 13:3-10.

Pelletti, Marco. 1989. Note al rilievo del Pantheon. Quaderni dell'Istituto di Storia dell'Architettura 13:10-8.

Rondelet, Antoine-Jean-Baptists. 1860. Étude sur la coupule du Pantheón de Rome. Paris: E. Lacroix.

Saalman, Howard. 1988. The Pantheon coffers: pattern and number. Architectura, Journal of the History of Architecture 18:121-122.

Scherer, Michael. 2009. A sustainable geodetic network for documenting and monitoring the Pantheon. In: The Pantheon in Rome. Contributions to the conference (Bern, November 9-12, 2006), Gerd Graßhoff, Michael Heinzelmann and Marcus Wäfler, eds. Bern: Bern Studies in the History and Philosophy of Science, pp. 215-22.

Taylor, Rabun. 2006. Los constructores romanos: un estudio sobre el proceso arquitectónico. Madrid: Akal.

Terenzio, Alberto. 1933. La restauration du Pantheon de Rome. Conservation de Monuments d'Art et d'Histoire: $280-85$.

Valenti, Graziano Maria. 2009. A computing model for the Pantheon's cupola: from the discrete to the continuous. the ideal continuous model. The Pantheon in Rome. Contributions to the conference (Bern, November 9-12, 2006), Gerd Graßhoff, Michael Heinzelmann and Marcus Wäfler, eds. Bern: Bern Studies in the History and Philosophy of Science, pp. 223-233.

Waddell, Gene. 2008. Creating the Pantheon: Design, materials, and construction. Roma: "L'ERMA" di Bretschneider.

Wilson Jones, Mark. 2000. Principles of Roman Architecture. New Haven: Yale University Press.

Licinia Aliberti is an architect from Roma Tre Universtity and holds a Ph.D. from the Technical University of Madrid with a research on geometry and construction of Pantheon and Roman domes. Her dissertation received a special mention in the Ph.D. award of the ETS of Architecture. She has been professor of geometry and graphical subjects at the School of Architecture of the European University of Madrid. At the present she teaches at the School of Design, Innovation and Technology of the University Camilo José Cela of Madrid. Her work focuses on contemporary metric restitution techniques and geometrical analysis of buildings, with a special interest on architectural heritage. She has been involved in the study and the graphical restitution of the ancient walls of Avila and the ancient walls of Santo Domingo de la Calzada (Spain) promoted from the Spanish Institute of Cultural Heritage. She participates in seminars and research activities both in Spain and Italy and she has written several studies for national and international publications. 
Miguel Ángel Alonso-Rodriguez is an architect and surveying engineer. He holds a Ph.D. in architecture; his dissertation focuses on the history of axonometry. He is Professor of Descriptive Geometry at the School of Architecture of the Polytechnical University of Madrid. He has lectured also at graduate courses on the history of axonometry and surveying at Madrid, at the Universidad Nacional Autónoma de Guadalajara (Mexico) and the Ecole Polytechnique d'Architecture et d'Urbanisme de Alger (Algerie). He has carried on surveys of historical buildings or archeological sites in Spain and Pompei both as the result of research grants, such as the ones included in Cantería renacentista en la catedral de Murcia or as a commissions from such key Spanish institutions as Patrimonio Nacional or the Real Academia de San Fernando.His research, focused on stereotomy and other issues concerned with spatial representation, is published regularly in refereed journals, international conferences. 